
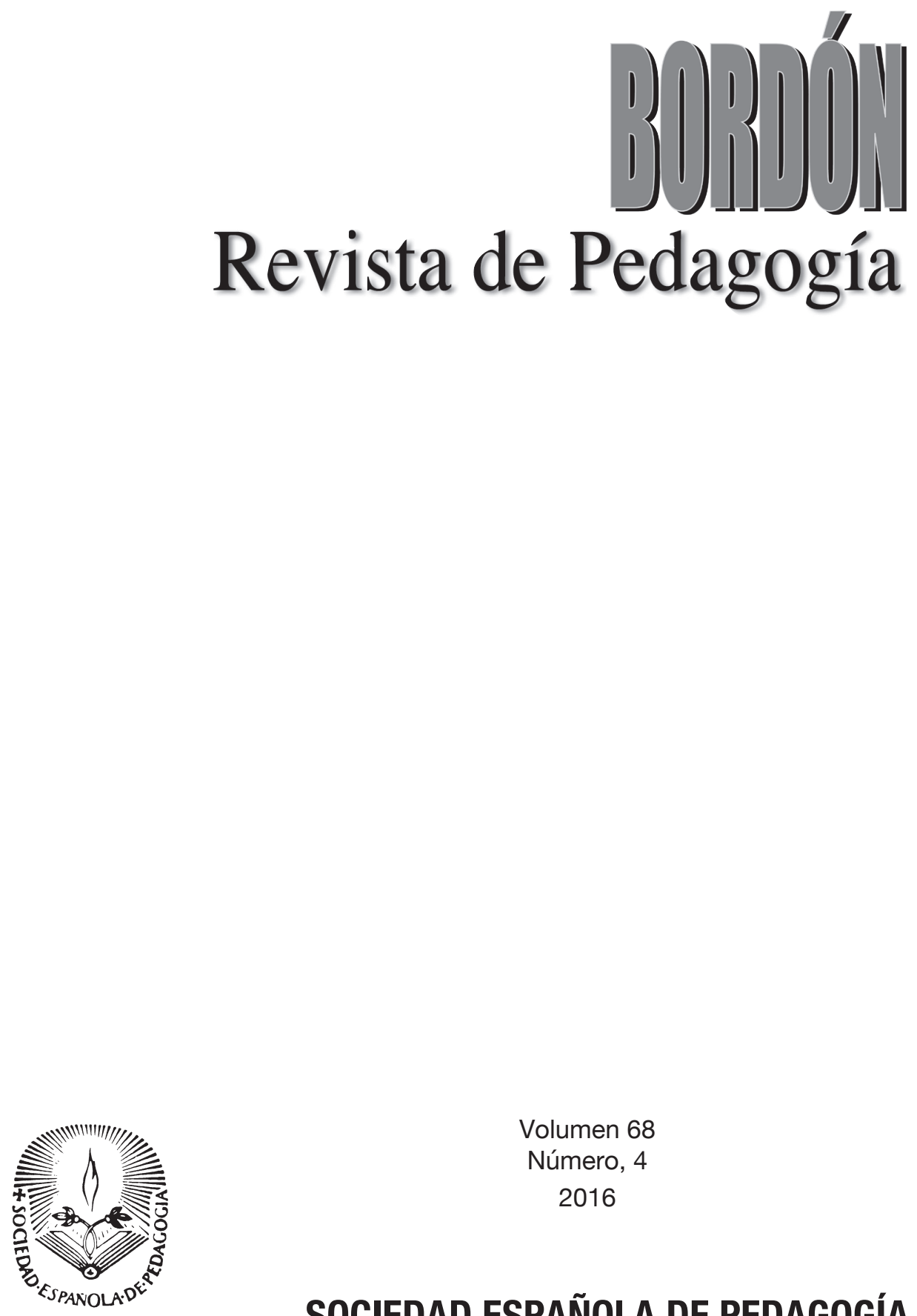

Volumen 68

Número, 4 2016 


\title{
PERCEPCIONES DEL PROFESORADO Y DE LOS PADRES DEL PACIENTE SOBRE LA PEDAGOGÍA HOSPITALARIA
}

\section{The perceptions of teachers and the patients' parents on hospital education}

\author{
ASCENSIÓN PALOMARES-RUIZY BELÉN SÁNCHEZ-NAVALÓN \\ Universidad de Castilla-La Mancha
}

DOI: 10.13042/Bordon.2016.38618

Fecha de recepción: 03/09/2015 - Fecha de aceptación: 23/06/2016

Autora de contacto / Corresponding Author: Ascensión Palomares-Ruiz. E-mail: ascension.palomares@uclm.es

Fecha de publicación online: 27/07/2016

INTRODUCCIÓN. La respuesta a las demandas que la sociedad plantea a la educación suele ser lenta e insuficiente, provocando discriminaciones, esencialmente, al alumnado que presenta problemas de salud. La pedagogía hospitalaria es una disciplina de flamante creación y su implantación no es igual en todos los países, por lo que investigar dicha realidad posibilita reflexionar y conocer el impacto que produce un ingreso hospitalario prolongado en los pacientes, sus familias y centros educativos de referencia. MÉTODO. Este trabajo pretende estudiar los beneficios e innovaciones que supone para los principales factores externos la pedagogía hospitalaria en las Unidades de Salud Mental, concretamente en la Unidad de Trastornos del Comportamiento Alimentario (UTCA) de un complejo hospitalario universitario que, por sus características, es un referente nacional. La investigación se desarrolla durante diez años bajo una metodología mixta. La muestra que se considera en el estudio está integrada por todos los padres de las alumnas que han sido atendidas en la UTCA $(\mathrm{N}=149)$ y por 149 docentes de los centros educativos de referencia. RESULTADOS. Los padres valoran las mejoras en el comportamiento y autoestima, y reconocen la ayuda facilitada por los miembros de la UTCA. Los docentes evalúan positivamente la información y coordinación con el Equipo de Atención Educativa Hospitalaria y Domiciliaria (EAEHD). Advierten que en el grupo-clase existe preocupación y apoyo hacia la compañera ingresada, y apenas cambian su metodología. Ambos grupos valoran positivamente su implicación durante el ingreso del paciente y, una vez superado, se incorpora en su nivel curricular sin grandes problemas. DISCUSIÓN. Se pone en evidencia la necesidad y efectividad de la unidad estudiada y se demanda una mayor implicación y formación del profesorado, así como el uso de las tecnologías de la información y la comunicación (TIC), para alcanzar una educación realmente inclusiva.

Palabras clave: Profesorado, Padres, Trastornos alimentarios, Niños hospitalizados, Tecnologías de la información, Educación no discriminatoria. 


\section{Introducción}

La implementación formal de la pedagogía hospitalaria comienza a principios del siglo XX. Su puesta en marcha, como señala García Álvarez (2013), varía en cada país, dependiendo de los gobiernos y de sus políticas educativas y sanitarias. Conocer de forma holística esta realidad nos permitirá reflexionar y debatir sobre el impacto que produce, en las familias y en los centros educativos de referencia, un ingreso hospitalario prolongado, así como la valoración que efectúan los propios protagonistas.

Sin pretender realizar un análisis detallado del desarrollo de la pedagogía hospitalaria en España, conviene subrayar los aspectos más significativos que la han conducido a la situación actual. En primer lugar, es preciso recordar que, en 1912, el Ministerio de Educación, reconociendo las buenas prácticas desarrolladas en otros países europeos y latinoamericanos, consideró la necesidad de instalar una "escuela", en el ambiente hospitalario (Casanova, 2007). Lentamente se empieza a reconocer el trabajo de los maestros en este medio, y en 1947 se instaura la primera escuela de forma regulada en el Hospital Clínico San Carlos de Madrid. Más adelante, en la década de los sesenta, una epidemia de poliomielitis, provoca largas hospitalizaciones de niños y niñas. Es el momento de plantearse la ayuda médica junto a la educativa.

En 1974 se abre el Hospital Nacional de Parapléjicos de Toledo con una filosofía innovadora que ofrece un enfoque integral a sus enfermos. Es importante destacar que se convierte en uno de los pocos hospitales públicos de España que, desde que se diseñó, contempla la existencia de este servicio de atención pedagógica.

Posteriormente, en 1981, otro caso grave sanitario de gran impacto social, el Síndrome Aceite Toxico (SAT), conocido como del "aceite de colza”, despierta la conciencia de las políticas educativas y, el 7 de abril, se publica la Ley de Integración Social del Minusválido (LISMI, 1982).
Poco después, en la década de los noventa, se promueve la inclusión educativa y la atención a la diversidad, apoyando y reforzando su implantación en un medio que no es el habitual. Con esta medida, las Administraciones pretenden garantizar que el alumnado enfermo pueda continuar su proceso educativo y mejorar las condiciones de estancia en el centro hospitalario (Muñoz Garrido, 2013).

Actualmente, en los hospitales se tiende a reducir el tiempo de los ingresos y la convalecencia se realiza, siempre que es posible, en el domicilio. Esta situación pone en marcha otra nueva modalidad dentro de la pedagogía hospitalaria, que es la atención domiciliaria. El profesorado se traslada al domicilio del alumnado convaleciente y sirve de puente de unión entre el centro educativo y el hogar (Grau, 2005). Por otro lado, el avance de las tecnologías de la información y la comunicación (TIC) permiten ampliar el contexto de la pedagogía hospitalaria y facilitan, tanto al paciente hospitalizado como al convaleciente en su domicilio, el contacto permanente con el mundo exterior, con su grupo-clase y sus amigos (Serrano y Prendes, 2015).

En las últimas décadas, se ha procurado hacer efectivo el derecho a la educación y así se presta un mayor interés a modalidades educativas como la educación hospitalaria, que históricamente habían presentado un desarrollo subsidiario (García Álvarez y Ruiz, 2014). Asimismo, se están introduciendo nuevas metodologías innovadoras, como el aprendizaje por proyectos (Poza y De la Blanca, 2014; Peirats y Granados, 2015) o el uso de videojuegos, en donde las emociones y el juego se conjugan y propician ambientes más motivadores para el desarrollo de contenidos curriculares (Guerra y Revuelta, 2015).

La atención hospitalaria y la domiciliaria se encuentran dentro del programa de atención a la diversidad, en el marco de la escuela inclusiva (García Álvarez, 2013). En consecuencia, la sociedad, las familias y los centros educativos 
precisan una información adecuada sobre esta disciplina para poder construir un futuro más esperanzador y conseguir una auténtica educación inclusiva. Sin embargo, apenas se encuentran estudios e investigaciones sobre la pedagogía hospitalaria. Igualmente, también escasean los trabajos sobre la utilidad de la Unidad de Trastornos del Comportamiento Alimentario (UTCA). No obstante, estos trastornos están aumentando progresivamente en todas las sociedades, resultando preocupante la "información" que prolifera en los medios de comunicación y en las redes sociales, brindando nuevos modelos con un cuerpo anormalmente delgado, que se asocia con el éxito y la belleza, provocando inseguridad y baja autoestima, entre otros problemas. Razonablemente, ante los Trastornos del Comportamiento Alimentario (TCA) se precisa una completa y coordinada atención, con la participación activa del entorno familiar y educativo. Consecuentemente, las políticas educativas intentan favorecer la atención a la diversidad en el contexto hospitalario, procurando que el alumnado internado tenga cubiertas sus necesidades educativas de la forma más normalizada posible.

Una vez revisadas las fuentes bibliográficas y el estado de la cuestión, es preciso conocer los principales factores humanos externos al paciente: los padres del alumnado ingresado y el profesorado del aula hospitalaria y de los centros educativos de referencia.

\section{Características de los padres del alumnado ingresado}

El colectivo de los padres es muy importante a la hora de trabajar la hospitalización y convalecencia de este alumnado. Por ello, como indican Bermúdez y Torío (2012), resulta fundamental conocer cómo vive la familia la enfermedad, cuya desestructuración suele ser proporcional a la gravedad y tiempo que se prolonga.
La comunicación que reciban los padres de los especialistas, y la que ellos transmitan al resto de la familia, debe ser continua y precisa. La respuesta de los padres, hermanos y familiares a la hospitalización del enfermo, como indican Lizasoáin y Ochoa (2003), es de vital importancia, ya que la familia es el núcleo básico en el que se apoyan las personas enfermas y su principal soporte psicológico durante todo el ingreso. En este sentido, Serradas (2003) expresa que la enfermedad constituye un hecho modificador frente a la rutina de lo cotidiano, y pone a prueba lo que, hasta ese momento, le era familiar y entrañable. Es una vivencia negativa sobreañadida al dolor, de la que no se posee experiencia previa y, lamentablemente, no suele recibirse la información precisa.

La alteración de los comportamientos, hábitos y ritmos de vida del alumnado por la enfermedad provoca cambios significativos que, por su intensidad, pueden causar conductas inadaptadas en un futuro, como trastornos de crecimiento, del sueño, del carácter, etc., factores que consiguen obstaculizar la vida normal de la familia. Incluso, llegan a provocar situaciones en las que los padres pierden la autoridad y tienen dificultades para actuar ante estas conductas inadaptadas y/o asociales (Polaino-Lorente, Abad, Martínez y Del Pozo, 2000).

Conviene indicar que, cuando la enfermedad es crónica, es posible que irrumpa en la familia como una agresión muy fuerte, al estar sometida a las constantes hospitalizaciones, se encuentra inmersa en el temor ante los procedimientos médicos, y sufre al apreciar cómo merma su vida familiar y social (López Naranjo y Fernández Castillo, 2006).

Los padres pueden padecer perturbaciones emocionales, como crisis de ansiedad o trastornos depresivos, al igual que puede afectar a otros miembros de la familia. Efectivamente, la persona enferma suele estar acompañada por algún miembro de su entorno que adquiere un significativo impacto psicológico, socioemocional y de 
salud, y lo va a sentir desde diferentes perspectivas, dependiendo de sus lazos familiares y de la empatía. En este sentido, se han estudiado las reacciones que puede tener la familia cuando conoce el diagnóstico, por lo general, se generan sentimientos muy intensos, de incredulidad, rabia, culpa, etc., que pueden llevar incluso al aislamiento (Lizasoáin, 2016).

Los hermanos, como especifican Espada y Grau (2012), experimentan sentimientos de culpa y se sienten desplazados, ya que suelen pensar que el hermano enfermo recibe más favores, lo que puede generar envidias entre ellos. En efecto, en ocasiones, los padres reducen su atención a los otros hijos y olvidan sus necesidades, esto a veces fomenta el que se formen coaliciones o exclusiones emocionales entre sus miembros, provocando un aumento de la frustración y una falta de comunicación, que pueden ocasionar serios problemas en el seno familiar.

Los abuelos, dadas las circunstancias de la sociedad actual, han cobrado un principal protagonismo en el cuidado de los nietos, al convertirse en agentes activos a la hora de atender y cuidar a la niña o al niño enfermos (López Naranjo y Fernández Castillo, 2006). Los abuelos pueden sufrir diferentes sentimientos (tristeza, incredulidad, culpabilidad, etc., pero, aun así, son de gran ayuda para la familia y llegan a convertirse en un pilar de tranquilidad y sosiego.

Los padres, elemento clave en el proceso de comunicación con la escuela, deben poseer un conocimiento correcto del estado en el que se encuentra su hijo o hija y ser encauzados por los docentes del aula hospitalaria para que asuman la responsabilidad de su educación y tratamiento. En este sentido, el objetivo de la pedagogía hospitalaria se centra en el apoyo al alumnado enfermo o convaleciente en su progreso educativo y a los familiares o personas que le rodean en su vida. Este servicio, a veces, solo lo conocen sus usuarios; incluso, hay personal sanitario en los hospitales que desconoce su existencia (Rosselló, De la Iglesia, Paz-Lourido y Verger, 2015).
Los profesionales de la salud deberían ser conscientes del papel que juega la educación en el desarrollo integral de las personas enfermas. Además, en el entorno próximo (médicos pediátricos, sanitarios, psicólogos, psiquiatras, terapeutas ocupacionales, las ONG, voluntariado, etc.), se precisa un ambiente de normalización; es decir, que el paciente se encuentre lo más cómodo posible, en unas condiciones lo más parecidas a las que llevaba en su vida habitual antes de estar enfermo. De ahí, la importancia de una buena coordinación entre todos los profesionales que atienden al paciente, con el fin de evitar interferencias o solapamientos que dificulten la labor educativa.

Consecuentemente, la pedagogía hospitalaria va, en la actualidad, más allá del trabajo educativo formal, y se preocupa de toda la población del ámbito hospitalario, facilitando el asesoramiento preciso para interactuar con el enfermo (Cárdenas y López Noguero, 2006). Por tanto, resulta esencial establecer unos cauces de comunicación adecuados, porque las emociones no van aisladas e interactúan como un nexo de identidad, conectando los pensamientos, juicios y creencias. Dan un gran significado a las experiencias que, la mayoría de las veces, no son positivas por falta de una comunicación apropiada (Yin y Lee, 2012). En este sentido, en algunos hospitales se está introduciendo la figura del educador social, cuya labor es atender las necesidades y problemas humano-sociales que se originan en el ámbito hospitalario.

\section{Coordinación del profesorado del aula hospitalaria y del centro educativo}

La coordinación del aula hospitalaria y/o de atención domiciliaria con el centro educativo de origen del paciente debe ser continua, e intentar que el centro esté lo más cercano al hospital y al hogar.

Desde hace dos décadas, las TIC se están convirtiendo en una herramienta muy eficaz por la 
rapidez con la que el profesorado del aula hospitalaria o de atención domiciliaria accede a la información que afecta al alumnado hospitalizado. Igualmente los padres deben implicarse en dicha coordinación, convirtiéndose en vehículos trasmisores, desarrollando una labor de mediación entre el centro educativo y el aula hospitalaria, y aportando información sobre sus características escolares y personales.

Es necesaria una coordinación del aula hospitalaria con el centro de referencia, si el alumnado tiene una estancia media o larga; es decir, más de 15 días de ingreso. Si el tiempo es menor, se realizan actividades lúdicas, de repaso de materias, trabajos propuestos por el docente del hospital, actos para conmemorar alguna festividad, colaborar con artículos o dibujos en la revista del aula hospitalaria, etc. Sin embargo, si el ingreso es superior a quince días, el profesorado del aula hospitalaria se pone en contacto con el centro, a través del Departamento de Orientación, exponiendo el estado del enfermo y especificando las actividades que puede realizar. A partir de este momento, es tutorizado por el docente del aula hospitalaria, formalizando los trabajos propuestos por el equipo docente (ED), hasta que reciba el alta y/o pueda incorporarse a su centro.

En las unidades de larga estancia, donde se acogen pacientes infantiles y juveniles, sobre todo, con los que padecen problemas de salud mental o enfermedades crónicas, la coordinación es muy parecida a la que se desarrolla en el aula hospitalaria convencional; pero, al tratarse de estancias prolongadas, existe una comunicación más fluida y, en casi todas las ocasiones, presencial. Si la permanencia se alarga y se aproximan las fechas de evaluación, los docentes de las unidades se desplazan al centro escolar, asisten a la sesión de evaluación e informan de los aspectos procedimentales y actitudinales, ya que los conceptuales son evaluados por el profesorado del centro a través de todas las actividades que ha realizado el alumno y que el profesor del aula hospitalaria ha enviado oportunamente.
Una vez que el enfermo es dado de alta, el tutor o el orientador del centro prepara su incorporación, para que sea recibido adecuadamente por sus compañeros. En el caso de que la patología haya deteriorado su aspecto físico o haya sufrido amputaciones se informa a todo el alumnado del cambio físico, y se le prepara para que no se produzcan situaciones o rechazos que puedan herir a la persona afectada que se incorpora o a sus compañeros.

Lógicamente, para que la coordinación sea eficaz, los centros de origen del alumnado hospitalizado o convaleciente deben cumplir los siguientes requisitos:

a) Remitir la documentación escolar precisa del alumnado con problemas de salud para que se pueda llevar a cabo la intervención educativa.

b) Facilitar la coordinación con el profesorado del aula hospitalaria en las actuaciones relacionadas con la evaluación y el seguimiento del alumnado enfermo atendido en la misma, considerando sus necesidades educativas y las posibles dificultades que pueda presentar.

c) Arbitrar procedimientos que faciliten la evaluación de dicho alumnado, teniendo en cuenta las necesidades educativas que manifiesten.

d) Colaborar con el diseño del plan de acogida para que el alumnado afectado sea bien recibido por su grupo-clase.

Resulta preciso subrayar que la coordinación desde las aulas hospitalarias con los centros de origen de los alumnos hospitalizados o convalecientes en sus domicilios debe regirse por la inclusión como principio fundamental de las políticas educativas. En la línea planteada por Lizasoáin (2016), también se considera la educación inclusiva como un proceso permanente, cuyo objetivo es ofrecer una educación de calidad para todos y todas, respetando la diversidad y las distintas necesidades y aptitudes, características y expectativas de aprendizaje de los estudiantes y de las comunidades educativas, y 
eliminando toda forma de discriminación. Además, la inclusión está relacionada con el acceso, la participación y logros de todo el alumnado, con especial énfasis en las personas que están en riesgo de ser marginadas, incluidas las que, por problemas de salud, no pueden asistir de forma regular a un centro educativo.

\section{Método}

El trabajo que se refleja en este artículo está contextualizado en una investigación más amplia, "El cambio social y educativo reflejado en la pedagogía hospitalaria de CLM", realizada por miembros del grupo de investigación EDUCALIDAD de la Universidad de Castilla-La Mancha, desde septiembre de 2003 hasta diciembre de 2015.

El contenido de la investigación que aquí se presenta es eminentemente educativo. Se ha tratado de analizar la importancia que asume el aula hospitalaria para los padres de los pacientes adolescentes y para el profesorado de los centros educativos de referencia, en dispositivos sanitarios que reciben pacientes en edad escolar durante ingresos prolongados. Se ha tomado la muestra sobre la Unidad de Trastornos del Comportamiento Alimentario (UTCA) del Hospital Universitario Nuestra Señora del Perpetuo Socorro de Albacete. En esta unidad se viene trabajando desde su creación, en 2002, con alumnado que presenta problemas de salud desde los 11 años a la edad adulta, y dentro de las dos posibles modalidades de ingreso (hospitalización completa y hospital de día), encontrándose ubicada en uno de los dos hospitales que forman el Complejo Hospitalario Universitario de Albacete. Desde su puesta en marcha, han desempañado su trabajo dos docentes que forman parte del Equipo de Atención Educativa Hospitalaria y Domiciliaria (EAEHD). Gracias a su actuación, la UTCA se ha convertido en un centro único en España y es un modelo de referencia a seguir para el resto del país.
El objetivo de la investigación que se refleja en este artículo es: conocer y analizar los posibles beneficios e innovaciones que aporta la pedagogía hospitalaria, dentro de las unidades de salud mental de larga estancia, según la percepción de los padres y del profesorado de los centros educativos de referencia de las alumnas ingresadas. Será importante saber cómo evalúan los padres el aula hospitalaria dentro de la UTCA, así como la opinión y el compromiso del profesorado de los centros de referencia. Asimismo, resultará interesante estudiar cómo ven la coordinación entre el EAEHD y los centros educativos (IES), igual que los posibles cambios que se perciben en el grupo-clase y ante la utilización de nuevas metodologías para el alumnado hospitalizado.

La muestra recogida es intencional y está integrada por los padres de 149 alumnas que han asistido al Aula hospitalaria durante los cursos 2003-2004 al 2012-2013 (de un total de 208 alumnas, es decir, la muestra comprende el $71,63 \%$ de los casos que fue posible localizar), y por 149 docentes de los Centros educativos de referencia que han trabajado con miembros del Aula hospitalaria. La muestra utilizada se formaliza con todos los datos obtenidos durante diez años. La constituyen padres cuyas hijas ingresadas en la UTCA tienen las siguientes características: son mujeres, con una media temporal de ingreso de 3 a 9 meses, edad entre 14 y 17 años, y cursan sus estudios mayoritariamente en centros públicos de Albacete capital y su provincia. Los docentes han sido elegidos al azar, en igual número que los padres localizados (149), con el requisito de que hayan colaborado con el profesorado del aula hospitalaria y conocer la dinámica de trabajo del EAEHD.

Todos los métodos con los que se lleva a cabo la investigación conducen a un enfoque sociocrítico y descriptivo de la situación del alumnado con problemas de salud, donde el conocimiento se va a construir por los intereses que parten de las necesidades de los dos grupos estudiados, y cuyas valoraciones servirán de base para propiciar una 
trasformación de la pedagogía hospitalaria. Por ello, no se entra en el dualismo de lo cuantitativo y lo cualitativo, ya que la investigación planteada no es solo un problema de métodos y técnicas, sino de conocimiento y de interpretación de la realidad para intentar mejorarla. Se combina el carácter cuantitativo con el cualitativo, pues interesa tanto el porcentaje numérico de las preguntas categorizadas, como los resultados de las respuestas abiertas y de las observaciones no sistemáticas realizadas durante un periodo de diez años, por ofrecer una información complementaria muy importante para la investigación.

El cuestionario es el principal instrumento de recogida de información utilizado y, dependiendo del grupo al que va dirigido, es diferente. Para alcanzar el objetivo del estudio se diseñó y validó un cuestionario elaborado ad hoc. Anteriormente se sometió a una aplicación piloto, en la que participó el alumnado que en ese momento se atendía en el aula y sus padres. De esta forma se corrigieron y aseguraron los aspectos que no estaban claros, la duración, el lenguaje, la forma de presentación y las instrucciones ofrecidas.
Dentro del profesorado se seleccionó un grupo al azar para que opinaran sobre la comprensión de las preguntas o sugiriesen alguna modificación. Además, se realizó un juicio de expertos, seleccionados entre profesionales del ámbito de las aulas hospitalarias y especialistas en investigación educativa, para valorar la relevancia, pertinencia y claridad de cada ítem del cuestionario. Las aportaciones de los expertos evidenciaron la alta relevancia de los ítems del cuestionario de cada grupo (alumnado, padres, profesorado), así como su adecuación.

Los cuestionarios contaban con diferentes tipos de preguntas de identificación, de opinión e incluso de observaciones y propuestas, con el fin de enriquecer al máximo el estudio y obtener una información lo más completa posible. En la tabla 1, se reflejan las características relevantes del instrumento utilizado en el grupo de padres. Seguidamente, en la tabla 2, se especifican las características del instrumento aplicado al profesorado. No se incluyen las que se refieren al grupo del alumnado, pues, como ya se ha indicado anteriormente, en este artículo solo se

TABLA 1. Características relevantes del instrumento utilizado en el grupo de los padres

Indicadores
Ítem Naturaleza

Respuesta

\begin{tabular}{llll} 
Información del aula & 1 & Identificación & Categorizada \\
\hline Afrontamiento familiar de la situación & 2 & Identificación & Categorizada \\
\hline Procedimiento de solicitud & 3 & Identificación & Categorizada \\
\hline $\begin{array}{l}\text { Mejora de la relación alumno/familia postalta } \\
\text { Mejora del rendimiento académico postalta }\end{array}$ & 4 & Identificación-opinión & Categorizada / abierta \\
\hline $\begin{array}{l}\text { Mayor acercamiento padres-alumnos (terreno } \\
\text { académico) }\end{array}$ & 6 & Identificación-opinión & Categorizada / abierta \\
\hline $\begin{array}{l}\text { Relación padres-IES-contenido } \\
\text { Dificultades Aula UTCA }\end{array}$ & 7 & Categorizada / abierta \\
\hline Beneficios de la experiencia & 8 & Identificación & Categorizada \\
\hline Recomendación a futuros pacientes & 9 & Información-opinión & Categorizada \\
\hline Observaciones y propuestas & 10 & Identificación & Categorizada / abierta \\
\hline
\end{tabular}

Fuente: elaboración propia. 
analizan las percepciones de los padres y del profesorado.

En los cuestionarios se debía responder a los ítems en una escala de 1 a 5 de tipo Likert. A partir del permiso del Comité Ético del SESCAM, empezaron a pasarse desde finales de abril hasta primeros de noviembre de 2014. Al participante solo se le daba una oportunidad para responder. De esta forma, los datos no se contaminaban por coacción o por sesgo favorable para el investigador. Se ha comprobado que la dimensión cualitativa reúne los criterios de rigor relacionados con la credibilidad de la investigación. Además, dicho rigor está garantizado en lo que afecta a la dimensión cuantitativa, la construcción del instrumento, el control de todo el proceso y la recogida de la información (Pérez Juste, García Llamas, Gil Pascual y Galán González, 2009). Los análisis descriptivos se han realizado mediante el programa estadístico SPSS (v.22.0.). Se puede afirmar, por tanto, que la investigación se ha llevado a cabo a través de un método mixto o ecléctico.

\section{Resultados}

A partir de los objetivos planteados, el análisis de los resultados ofrece una visión global de la situación, según las opiniones vertidas por los participantes en la investigación. Resulta interesante destacar que en el colectivo de los padres, de los 73 que respondieron al cuestionario, el $78,87 \%$ afirma que el conocimiento del aula lo recibieron en la propia unidad y un $9,86 \%$ indica que supo de su existencia en el centro educativo.

En cuanto a si mejoró la relación paterno-filial después del ingreso y por qué, el 79,45\% responde afirmativamente. En cambio, el 21,55\% expresa no haber observado cambios significativos después del ingreso. Las causas que han propiciado la mejora en las relaciones, como se puede comprobar en el gráfico 1, según el 65\% se reflejan en su comportamiento; sin embargo, el $11 \%$ indica que la relación era buena antes del ingreso; el 10\% manifiesta que después del ingreso empeora; el $2 \%$ valora que la mejora

TABla 2. Características relevantes del instrumento utilizado en el grupo del profesorado

\begin{tabular}{lcll} 
Indicadores & Ítem & Naturaleza & Respuesta \\
\hline $\begin{array}{l}\text { Experiencia anterior EAEHD } \\
\text { Tiempo de experiencia }\end{array}$ & 1 & Identificación & Categorizada \\
\hline $\begin{array}{l}\text { Formación / información sobre este tipo de } \\
\text { alumnos }\end{array}$ & 3 & Identificación / información & Categorizada \\
\hline Actitud de los padres & 4 & Identificación & Categorizada \\
\hline $\begin{array}{l}\text { Dificultades en la implantación del aula } \\
\text { Clima del aula centro de referencia }\end{array}$ & 5 & Identificación / información & Categorizada / abierta \\
\hline $\begin{array}{l}\text { Interrupción de la rutina de clase } \\
\text { Utilización de nuevas tecnologías (TIC) }\end{array}$ & 7 & Identificación / información & Categorizada / abierta \\
\hline $\begin{array}{l}\text { Beneficios para el aula de las nuevas tecnologías } \\
\text { (TIC) }\end{array}$ & 8 & Identificación / opinión & Categorizada/ abierta \\
\hline \begin{tabular}{l} 
Mejoras para el aula de la UTCA \\
\hline Observaciones
\end{tabular} & 10 & Identificación / información & Categorizada / abierta \\
\hline
\end{tabular}

Fuente: elaboración propia. 
Gráfico 1. Causas de la mejoría en las relaciones paterno-filiales

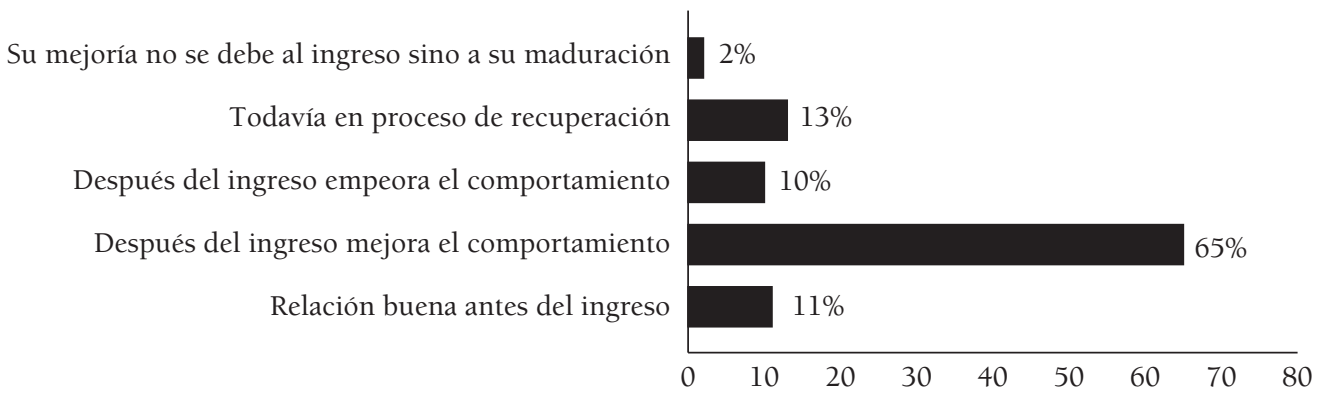

Fuente: elaboración propia.

depende de su maduración, y el 13\% informa que todavía está en proceso de recuperación.

Respecto a si existió un progreso en el rendimiento académico, el $42 \%$ indica que ha encontrado cambios positivos significativos en su hija después del ingreso y que su paso por la unidad ha sido muy favorable. Sin embargo, como puede observarse en el gráfico 2 , en el $58 \%$ no se advierten cambios (el 12\% alega que psicológicamente no ha avanzado, el 23\% manifiesta que el rendimiento académico era antes bueno, el $11 \%$ indica que el estudio le provocó más ansiedad y el 12\% le da más importancia al tema académico que a la salud).

Como puede comprobarse en el gráfico 3, los usuarios más directos del servicio, como son las alumnas y sus familias, realizan una valoración muy positiva del aula de la UTCA, especialmente por la ayuda para la organización del tiempo de estudio y no tener que repetir curso, y en colaborar para incorporarse a su vida con toda normalidad. El porcentaje es muy alto en este grupo (90\%), por lo que se puede considerar la labor del aula como muy positiva. Sin embargo, el 10\% afirma no observar cambios en la actitud de la alumna ante su negativa a estudiar.

En cuanto al colectivo de docentes del centro de referencia, a la pregunta del tiempo medio de experiencia con el EAEHD han contestado el 91\% de los participantes. En este sentido, la media es de 10,14 meses, aunque el 44\% señala que su experiencia es superior a un año. Ante la pregunta de quién le informó sobre esta modalidad

\section{GrÁfICo 2. Influencia de la unidad en el rendimiento académico}

Más importancia al estudio que a la salud

Con el ingreso se dio cuenta de la importancia de los estudios y recuperó la autoestima y la responsabilidad Psicológicamente no ha avanzado

El estudio le provocó más ansiedad y su incorporación al IES no lo ha facilitado. Problema entre iguales El rendimiento antes era bueno

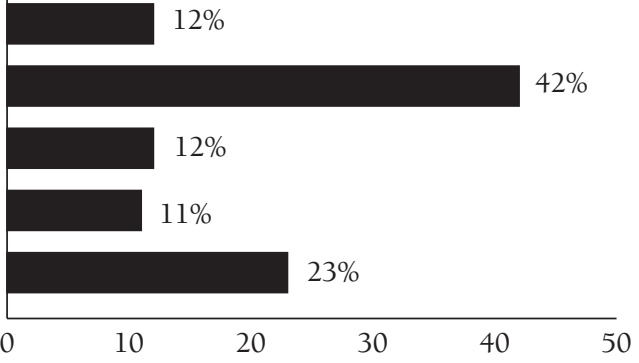

Fuente: elaboración propia. 
GrÁFICO 3. Valoración de la ayuda prestada por el aula de la UTCA

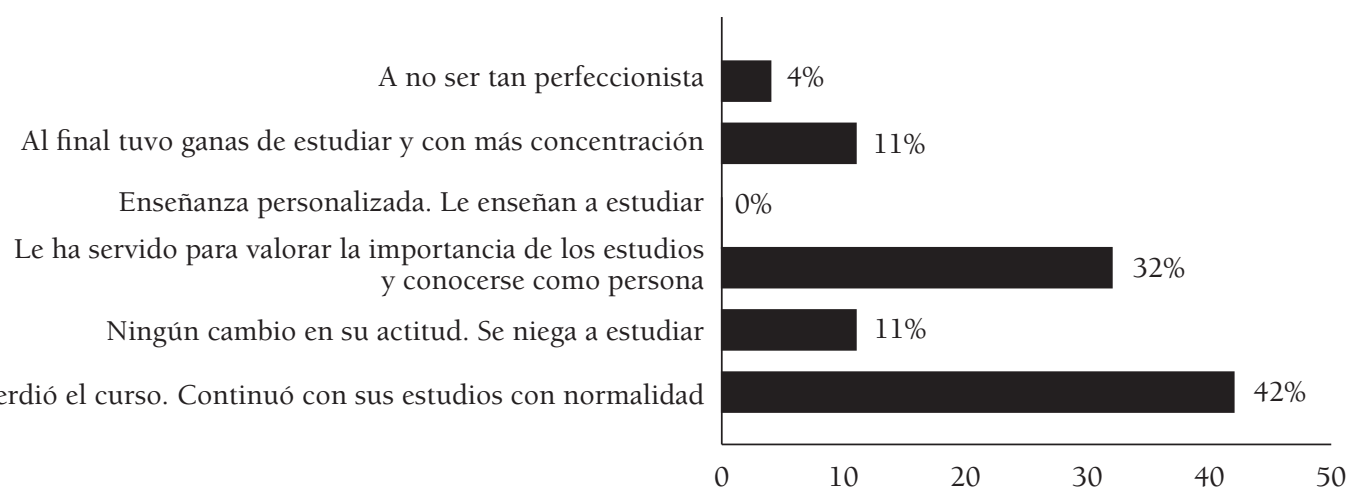

Fuente: elaboración propia.

de docencia, el 43\% del profesorado indica que la ha recibido del EAEHD. Ninguno, dentro de la atención a la diversidad, posee formación específica sobre este tipo de alumnado. Es importante destacar que el 54\% indica que no le ha supuesto mayor dificultad establecer las coordinaciones con los miembros de la unidad.

Después de conocer el ingreso de la alumna, como puede observarse en el gráfico 4 , el clima de la clase es de preocupación y apoyo (63\%),

\section{Gráfico 4. Clima de la clase tras el ingreso de la alumna}

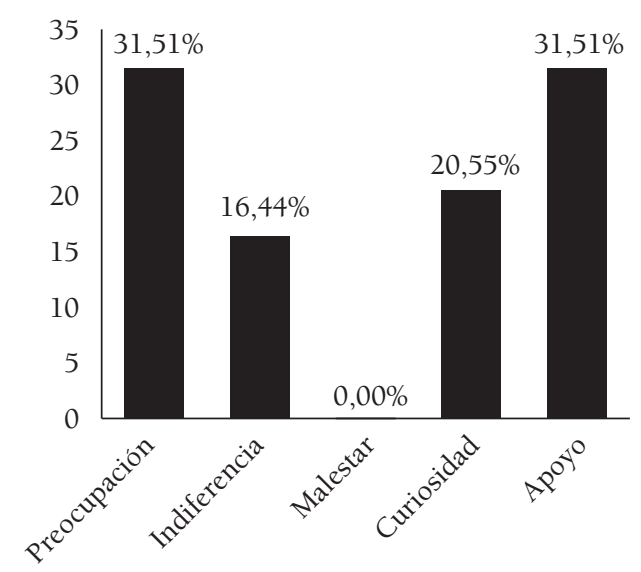

Fuente: elaboración propia. aunque también existe un 16,44\% de indiferencia por parte de sus compañeros y un $20,55 \%$ muestra curiosidad.

Como resultado de la interrupción de la normalidad de la clase por parte del EAEHD, el $73,08 \%$ del profesorado reconoce que no se han producido interrupciones significativas, $y$ la organización y coordinación con los miembros del aula hospitalaria ha sido un proceso sencillo (gráfico 5).

\section{GrÁFICO 5. Valoración del profesorado sobre una posible interrupción en las clases}

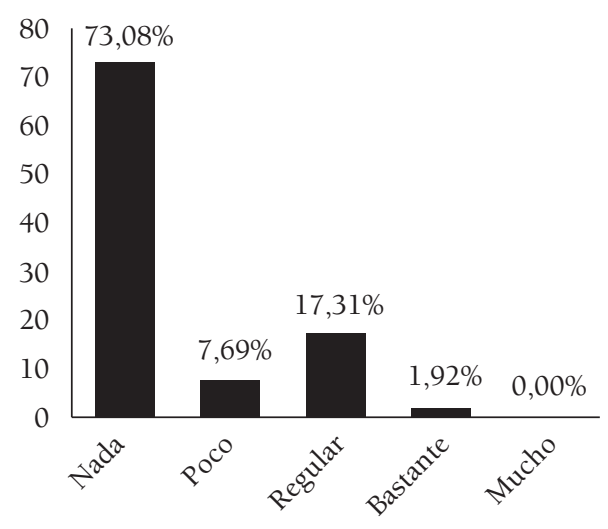

Fuente: elaboración propia. 
Es importante reflexionar sobre los efectos de la posible interrupción en la dinámica normal de aula del centro de referencia cuando la alumna llega al grupo-clase y tiene que adaptarse al ritmo normalizado del resto de sus compañeros. Como puede observarse en el gráfico 6 , el $38 \%$ del profesorado expresa que no se han presentado problemas en la incorporación. Por ello se cumple el objetivo más importante del aula de la UTCA: que no existan complicaciones en la incorporación del alumnado con problemas de salud a su centro educativo.

Si nos centramos en el establecimiento de nuevas propuestas metodológicas que benefician al alumnado hospitalizado, del 91\% que responde, un 56\% afirma haber modificado poco o nada su metodología. En cambio, un $35 \%$ manifiesta que ha sido preciso cambiarla. Entre los posibles cambios que ha realizado el profesorado del centro destaca la preparación anticipada del material, por tener que enviárselo al profesorado del aula hospitalaria. Asimismo, como se justifica en el gráfico 7 , se solicita que todo el material se remita de forma telemática para una mejor coordinación.

Sobre la valoración que realiza el profesorado acerca de los cambios realizados en la metodología, como se muestra en el gráfico 8, el 43\% expresa que dichos cambios estimulan la atención del alumnado, el 22\% considera que

\section{Gráfico 6. Posibles problemas en la incorporación al centro}

Ritmo paralelo al llevado en el centro por la coordinación EAEHD/ED

Al principio, ritmo más lento que el resto de compañeros

La parte teórica sin problemas, la práctica con dificultades

Ningún problema en la incorporación

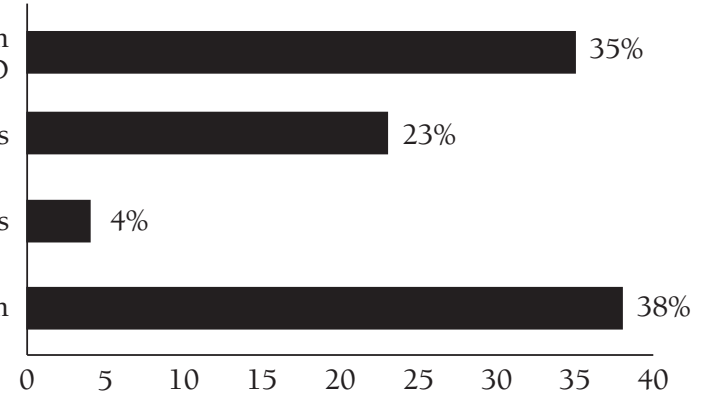

Fuente: elaboración propia.

\section{GráfICo 7. Cambios en la metodología utilizada}

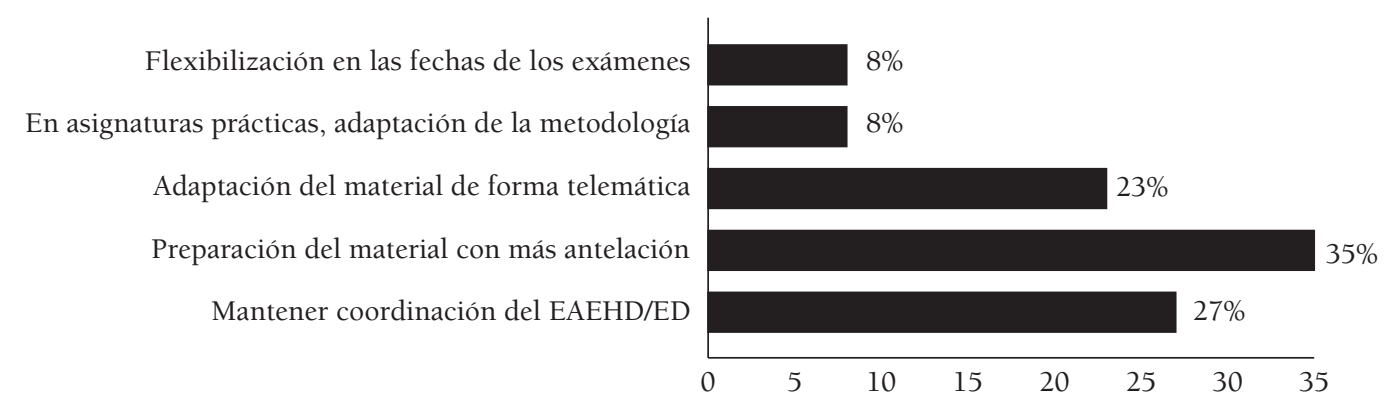

Fuente: elaboración propia. 


\section{GrÁfICo 8. Condicionantes que favorecen el cambio de metodología}

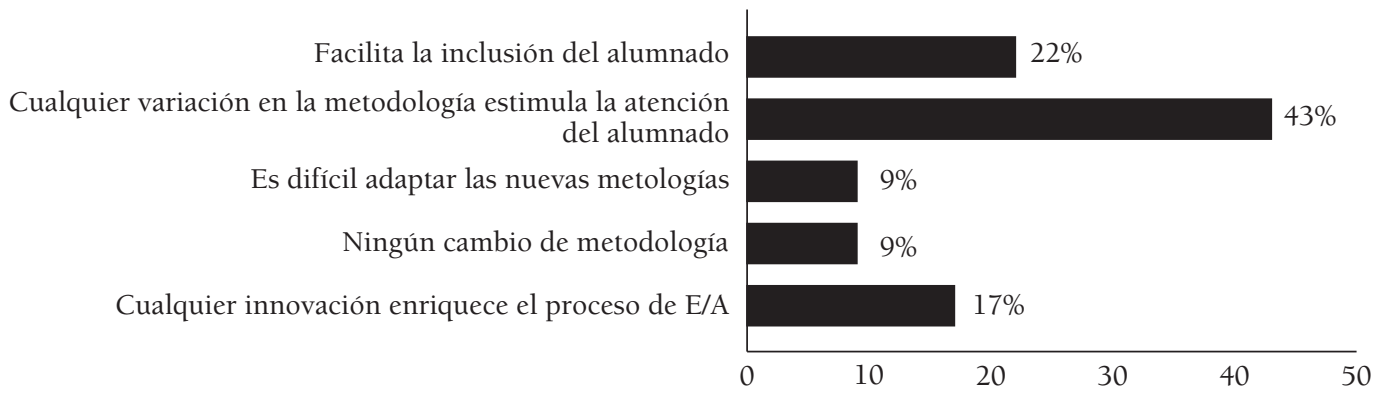

Fuente: elaboración propia.

\section{Gráfico 9. Mejoras que plantea el profesorado de los centros educativos}

Contacto visual con el alumnado a través de los medios telemáticos

Elevada ratio y pocos docentes en el EAEHD

Más información del EAEHD del avance del alumnado

Coordinación EAEHD/ED aceptable

Mejor coordinación EAEHD/Centro referencia

Este servicio mejora la calidad del sistema educativo

facilitan la inclusión educativa, el 17\% afirma que cualquier cambio enriquece el proceso de enseñanza-aprendizaje y el 18\% valora complicado introducir cambios metodológicos o, simplemente, no los han realizado.

Respecto a si establecerían algunas mejora en el aula hospitalaria, como se expresa en el gráfico 9, el 18\% del profesorado demanda más información sobre el estado de salud de la alumna ingresada, el 59\% valora como aceptable la coordinación EAEHD-ED, el 12\% alega que este tipo de servicio mejora la calidad del sistema educativo y el $12 \%$ opina que la carga de trabajo es muy elevada.

Fuente: elaboración propia.
$0 \%$

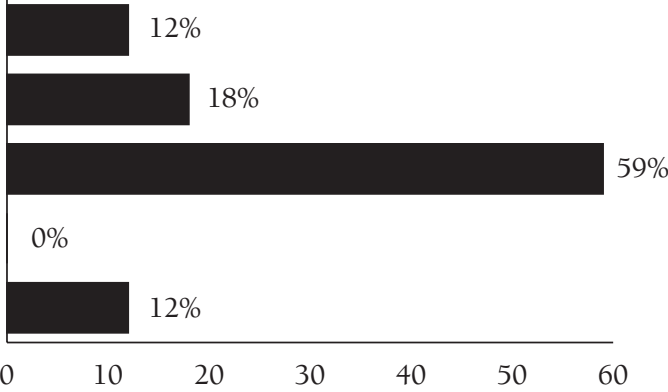

En la investigación se ha realizado una relación biunívoca entre padres y profesorado del centro educativo para comparar sus valoraciones. En la pregunta de cómo es la actitud de los padres hacia el centro, como se refleja en el gráfico 10 , el $71 \%$ del profesorado exterioriza que es muy buena y con un alto grado de implicación. Por otro lado, un 18\% muestra una actitud distante, que podría estar motivada por la situación familiar que está viviendo.

Analizados los resultados globalmente, se evidencia que existe una buena relación entre los padres de las alumnas con problemas de salud y el profesorado del centro educativo. Se valora 


\section{Gráfico 10. Actitud de los padres hacia el centro}

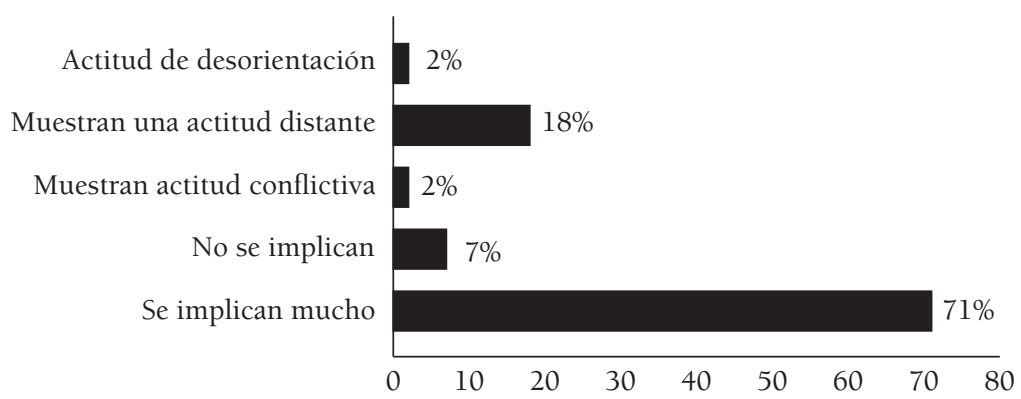

Fuente: elaboración propia.

muy positivamente el trabajo del EAEHD y lo consideran un servicio imprescindible dentro de las unidades de salud mental. Si nos centramos en el colectivo de los padres, se comprueba que les resultó fácil la tramitación para la asistencia al aula y que la información la recibieron en la propia unidad. Es oportuno resaltar las dificultades que encontraron por la falta de concentración de su hija o por problemas de coordinación con el centro, ajenos a las buenas prácticas del aula hospitalaria. Asimismo, consideran que el afrontamiento familiar ante la enfermedad fue de apoyo y de aceptación de la realidad, y que después del ingreso se generó una mejor relación padres-hijas que les ayudó a valorar lo que poseían y dar más importancia a los estudios.

El colectivo docente ha sido el menos implicado en esta investigación, posiblemente por falta de formación o interés sobre este tema. Los que han tenido experiencias con otros profesionales del EAEHD se han centrado en patologías como cirugía, oftalmología, Unidad de Salud Mental Infanto-Juvenil (USMIJ) y oncología. Esta última patología ocupa gran parte del horario de los docentes del EAEHD, el profesorado de los centros educativos indica que ha recibido información sobre este tipo de alumnado a través de las reuniones de coordinación con el profesorado del EAEHD, seguido por el Departamento de Orientación del centro. Sin embargo, es llamativa la falta de información y de formación del profesorado para poder trabajar de forma apropiada.

Los padres valoran mayoritariamente $(86,11 \%)$ que la actitud del profesorado del centro es buena o muy buena. El profesorado también señala de manera muy positiva, en un $71 \%$, la implicación de los padres. De estos resultados se deduce que existe una buena relación entre los padres y los docentes. Respecto a la valoración general del aula hospitalaria en su conjunto, así como las observaciones que de forma voluntaria se han producido, resultan de gran interés algunas manifestaciones como las siguientes: "Gracias por el apoyo y paciencia que tienen con los alumnos", "Se debe difundir en los Institutos de Educación Secundaria (IES) este tipo de patologías", "Valoración muy positiva de todo el equipo terapéutico, con especial mención al aula hospitalaria", "Se debe conocer más la metodología del EAEHD, a través de charlas informativas", "Estas aulas son necesarias en el siglo XXI, pero hay que dotarlas de personal y medios", etc.

\section{Discusión}

Se ha llegado a la conclusión, al igual que en otras investigaciones (García Álvarez, 2013; Muñoz Garrido, 2013; Lizasoáin, 2016), que las valoraciones de los padres y del profesorado del centro educativo de referencia son muy 
positivas, lo que anima a seguir trabajando y corrigiendo aquellos aspectos negativos que se extraen de esta investigación. Resulta ineludible que las respuestas educativas al alumnado con problemas de salud deben considerar a la persona de forma global, por lo que es necesario que se planifiquen y ejecuten desde un modelo interdisciplinar.

Este artículo se ha centrado en el estudio de las percepciones de los principales factores humanos externos al paciente; sin embargo, es preciso indicar que el $90 \%$ de las alumnas atendidas en el aula hospitalaria la consideran un recurso eficaz y positivo. Solo un 10\% no la recomendarían a otras pacientes, por existir una desvinculación con sus compañeros y compañeras. En este sentido, para superar la citada desvinculación, hay que incidir en la importancia de las TIC, con el fin de acercar el alumnado a su centro y aula.

De acuerdo con los resultados, es importante requerir a las personas que trabajan con la infancia y adolescencia que asuman su responsabilidad para que los menores que sufren alguna patología sean atendidos adecuadamente y se potencie su capacidad de desarrollo y su bienestar. Así, se facilitará su completa integración en el medio familiar, social y escolar, y su autonomía personal. De forma más exhaustiva, las mejoras que se proponen son las siguientes:

a) Una mayor formación psicopedagógica y concienciación del profesorado de los centros educativos de referencia (IES), para que se mejore la respuesta educativa al alumnado que presenta problemas de salud.

b) Impregnar a la sociedad con una correcta información sobre la necesidad de que el derecho a la educación sea una realidad para todas las personas, sin ningún tipo de exclusión.

c) Dotar de medios materiales y personales para que, según la problemática de cada niño o niña, se pueda garantizar una educación inclusiva. Para ello, en las aulas con alumnado que presente problemas de salud, habría que bajar las ratios y reducir las horas lectivas del profesorado además de disponer de los especialistas precisos.

d) Potenciar la formación y motivación del profesorado sobre medios telemáticos, con el fin de optimizar su utilización.

e) Mejorar la dotación de medios informáticos, independientes de la intranet del hospital, para que el alumnado con problemas de salud pueda participar en sus clases del IES vía telemática. Como indica Gómez Nicholls (2015), el avance tecnológico supera la capacidad de respuesta de las instituciones, por lo que es necesario un gran esfuerzo de actualización y mejora en este campo.

El análisis de la información obtenida evidencia que este trabajo puede servir para abrir nuevas vías de debate para una mejora de la pedagogía hospitalaria en España y, especialmente, en Castilla-La Mancha. Al igual que concluyen otras investigaciones como las realizadas por Muñoz Garrido (2013), habría que destacar la necesaria complementación y coordinación de las funciones educativas de la familia y la escuela para abordar las "modas" del entorno social, afrontando una formación integral de la persona y una educación para la salud desde edad temprana.

Asimismo, resultan incuestionables las puertas que abren las tecnologías de la información y la comunicación (TIC) en estas modalidades de educación, como ocurre desde hace décadas en los países del norte de Europa. En esta línea, hay que hacer un llamamiento a los responsables políticos para que regulen una adecuada información y formación de todos los implicados en la educación y la sanidad, con los recursos precisos que posibiliten ofrecer a todo el alumnado una auténtica educación inclusiva, sin ningún tipo de discriminación. 
Percepciones del profesorado y de los padres del paciente sobre la pedagogía hospitalaria

\section{Referencias bibliográficas}

Bermúdez, M. ${ }^{a}$ T., y Torío, S. (2012). Educación informal y ocio juvenil. La percepción de las familias ante la animación hospitalaria: estudio realizado en el hospital materno-infantil de Oviedo. Pedagogía Social. Revista Interuniversitaria, 20, 223-242.

Cárdenas, R., y López Noguero, F. (2006). Hacia la construcción de un modelo social de la Pedagogía Hospitalaria. Pedagogía Social. Revista Interuniversitaria, 12, 59-70.

Casanova, M. a A. (dir.) (2007). Atención educativa al alumnado enfermo en la Comunidad de Madrid. Madrid: Consejería de Educación.

Espada, M. C., y Grau, C. (2012). Estrategias de afrontamiento en padres de niños con cáncer. Psicooncología. Revistas Científicas Complutenses, 9 (1), 25-40.

García Álvarez, A. (2013). Escuelas Hospitalarias en España, Suecia y Argentina: Evolución y situación contemporánea de las instituciones comprometidas con la educación del niño y adolescente en situación de enfermedad (tesis doctoral). Universidad Autónoma de Madrid. Recuperada de https:// repositorio.uam.es/.../9114/48095_garcia_alvarez_antonio.pdf

García Álvarez, A., y Ruiz, G. (2014). El derecho a la educación y la educación para la diversidad: el caso de las escuelas y aulas hospitalarias en Europa. Journal of Supranational Policies of Education, 2, 72-92.

Gómez Nicholls, J. F. (2015). Soluciones para optimizar los procesos de aprendizaje en el ámbito hospitalario. Reposital. Recuperado de http://hdl.handle.net/123456789/3775.

Grau, C. (2005). La atención educativa de las necesidades educativas especiales de los niños enfermos de cáncer. Bordón. Revista de Pedagogía, 57 (1), 47-58.

Guerra, J., y Revuelta, F. I. (2015). Videojuegos precursores de emociones positivas: propuesta metodológica con Minecraft en el Aula Hospitalaria. International Journal of Educational Research and Innovation (IJERI), 3 (1), 105-120.

Ley 13/1982 de Integración Social del Minusválido (LISMI), de 7 de abril. Madrid: BOE del 30. Recuperado de https://www.boe.es/buscar/doc.php?id=BOE-A-1982-9983.

Lizasoáin, O., y Ochoa, B. (2003). Repercusiones de la hospitalización pediátrica en el niño enfermo. Osasunaz, 5, 75-85.

Lizasoáin, O. (2016). Pedagogía hospitalaria. Guía para la atención psicoeducativa del alumno enfermo. Madrid: Editorial Sintesis.

López Naranjo, I., y Fernández Castillo, A. (2006). Hospitalización infantil y atención psicoeducativa en contextos excepcionales de aprendizaje. Revista de Educación, 341, 553-577.

Muñoz Garrido, V. (2013). Pedagogía Hospitalaria y Resiliencia (Tesis doctoral). Universidad Complutense de Madrid. Recuperada de http://eprints.ucm.es/18133/1/T34246.pdf

Peirats, J., y Granados, J. (2015). Las unidades pedagógicas hospitalarias y el aprendizaje por proyectos de trabajo. Aula de Encuentro, 17 (1), 187-211.

Pérez Juste, R., García Llamas, J. L., Gil Pascual, J. A., y Galán González, A. (2009). Estadística aplicada a la educación. Madrid: Pearson Prentice Hall-UNED.

Polaino-Lorente, A., Abad, M., Martínez, P., y Del Pozo, A. (2000) ¿Qué puede hacer el médico por la familia del enfermo? El impacto del enfermo en la familia. Pamplona: Ediciones RIALP.

Poza, V., y De la Blanca, S. (2014). El desafío de la escuela inclusiva a través de los proyectos de trabajo y del método científico en un aula de primaria. Aula de Encuentro, 16 (2), 4-41.

Rosselló, M. R., De la Iglesia, B., Paz-Lourido, B., y Verger, S. (2015). Necesidades de formación psicopedagógica para la atención de niños con enfermedad crónica: percepciones de enfermería hospitalaria. Journal of School of Nursing, 49 (1), 37-43. Doi: 10.1590/S0080-623420150000100005

Serradas, M. (2003). La Pedagogía Hospitalaria y el niño enfermo: Un aspecto más en la intervención socio-familiar. Revista de Pedagogía, 24 (71), 447-468. 
Serrano, J. L., y Prendes, M. P. (2015). Integración de TIC en aulas hospitalarias como recursos para la mejora de los procesos educativos. ESE. Estudios sobre Educación, 28, 187-210.

Yin, H. B., y Lee, J. C. (2012). Be passionate, but be rational as well: Emotional rules for Chinese teacher's work. Teaching and Teacher Education, 28, 56-65.

\section{Abstract}

The perceptions of teachers and the patients' parents on hospital education

INTRODUCTION. The answer to the demands posed by society in regards to education is often slow and inadequate, causing discrimination, mainly to enrolled students with health-related problems. Hospital Pedagogy is a newly created discipline and its implementation is not the same in all countries. Therefore, to investigate this reality allows us to reflect and understand the impact that a lengthy hospitalization has on patients, their families and their educational centres of reference. METHOD. This work aims to study the benefits and innovations Mental Health Units of Hospital Pedagogy provide to the main external factors, particularly in the Eating Disorder Unit (UTCA) of a University Hospital which is, by its nature, a national benchmark. The research has been carried out for ten years using a mixed methodology. The sample considered in this study is composed by the students' attending the UTCA $(\mathrm{N}=149)$ and by 149 teachers working at the reference educational institution. RESULTS. Parents value the improvements in behaviour and selfesteem and appreciate the assistance provided by members of the UTCA. Teachers positively value the information received and coordination with the educational hospital and home care team, stressing that in the class-group there is concern and support towards the admitted classmate, making few changes in their methodology. Both groups recognise that their involvement has been good during the process of patient admission and once the the situation is overcome, patients are reincorporated in their curricular level without any major problems. DISCUSSION. The necessity and effectiveness of the studied Unit is obvious and a greater teacher involvement and training is required, as well as the use of Information and Communications Technology (ICT) to truly achieve an inclusive education.

Keywords: Teachers, Parents, Eating Disorders, Hospitalized Children, Information Technology, Nondiscriminatory Education.

\section{Résumé}

Perceptions du professorat et des parents du patient sur l'enseignement en milieu hospitalier

INTRODUCTION. La réponse aux demandes soumises par la société à l'éducation est en général lente et insuffisante, ce qui provoque des discriminations, essentiellement aux effectifs scolaires qui présentent des problèmes de santé. La Pédagogie Hospitalière est une toute nouvelle discipline et sa mise en ouvre diffère selon les pays, de sorte qu'étudier cette réalité permet de réfléchir et de connaître l'impact qu'une hospitalisation prolongée produit chez les patients, leurs familles et les établissements scolaires de référence. MÉTHODE. Cette étude a pour but d'étudier les bénéfices et les innovations de la Pédagogie Hospitalière sur les principaux facteurs externes dans les Services de Santé Mentale, concrètement dans l'Unité des Troubles du Comportement 
Alimentaire (UTCA) d'un Centre Hospitalier Universitaire qui, par ses caractéristiques, est un référent national. La recherche a été développée tout au long de dix années avec une méthodologie mixte. L'échantillon pris en compte dans cette étude comprend tous les parents des collégiennes ayant subi une modalité d'hospitalisation ( $\mathrm{N}=149)$ et 149 professeurs de l'établissement de référence. RÉSULTATS. Les parents apprécient les améliorations dans le comportement et l'estime de soi, en reconnaissant la valeur de l'aide fournie par les membres de l'UTCA. Les professeurs saluent la possibilité de coordination et l'information fournie par l'Équipe d'Attention Educative Hospitalière et à Domicile (EAEHD), et constatent la préoccupation et l'appui du groupe-classe envers le camarade hospitalisé, et changent à peine leur méthodologie. Les deux groupes évaluent positivement leur implication durant le processus d'hospitalisation du patient et, une fois celui-ci surmontée, le patient s'incorpore à son niveau d'études scolaires sans grand problème. DISCUSSION. La nécessité et l'effectivité de l'Unité étudiée sont mises en évidence. Une plus grande implication et formation du professorat sont demandées, ainsi que l'usage des Technologies de l'Information et de la Communication (TIC) pour parvenir à une éducation réellement inclusive.

Mots clés: Professeurs, Parents, Troubles alimentaires, Enfants hospitalisés, Technologie de l'Information, Éducation sans discrimination.

\section{Perfil profesional de las autoras}

\section{Ascensión Palomares-Ruiz (autora de contacto)}

Doctora en Filosofía y Ciencias de la Educación. Catedrática de Didáctica y Organización Escolar y coordinadora del Departamento de Pedagogía en la Facultad de Educación de Albacete (España). Directora del grupo de investigación EDUCALIDAD. Ejerce sus funciones docentes, de investigación y gestión en la UCLM y la UNED. Su perfil docente e investigador se centra en educación inclusiva, liderazgo y calidad de la educación, derechos humanos, prácticum y pedagogía hospitalaria. Correo electrónico de contacto: ascension.palomares@uclm.es

Dirección para la correspondencia: Facultad de Educación de Albacete. Universidad de Castilla-La Mancha. Pza. Universidad, nº 3. 02071 Albacete, España.

\section{Belén Sánchez-Navalón}

Doctora en Educación. Es profesora en la UCLM y miembro del Equipo de Atención Educativa Hospitalaria y Domiciliaria (EAEHD) de Albacete (España). Pertenece al grupo de investigación EDUCALIDAD. Su perfil docente e investigador se centra en la pedagogía hospitalaria y la educación inclusiva.

Correo electrónico de contacto: bsanvalon1@gmail.com. 